\title{
Die SIP der Stadt Luzern als Akteurin der vermitteln- den Sozialarbeit
}

Arbeitsfeld Intervention und Prävention im öffentlichen Raum

\section{Christina Rubin ${ }^{1}$, Lucia Sidler ${ }^{2}$}

\section{Einleitung}

Im Jahre 2005 formulierte der Stadtrat von Luzern Folgendes: «Die politische Strategie der Stadt Luzern ist es, eine SIP zu bilden, damit es in der Stadt Luzern zu keiner erneuten Bildung einer offenen Drogenszene kommt. Auf der anderen Seite soll der öffentliche Raum von allen Bevölkerungsgruppen genutzt werden können. Einer gesellschaftlichen Spaltung von ökonomisch und sozial verschieden starken Bevölkerungsgruppen soll entgegengewirkt werden» (Stadt Luzern 2009: 6).

Wie ist Luzern diesen Auftrag angegangen und wo steht die Stadt heute? Gedanken und Erfahrungen dazu von zwei SIP-Mitarbeiterinnen.

Die Soziale Arbeit als Profession insgesamt und die aufsuchende Sozialarbeit im Bereich Suchthilfe im Speziellen erfuhren in den letzten Jahrzehnten eine starke Spezialisierung. Die Soziale Arbeit etablierte sich in Bereichen, die zuvor nicht existierten oder die von anderen Professionen abgedeckt worden waren. Die Institution Sicherheit Intervention Prävention (SIP) der Stadt Luzern ${ }^{3}$ ist ein exemplarisches Beispiel für diese Entwicklung.

Die SIP war zu Beginn überwiegend regulativ in der Stadt unterwegs und war sich zu wenig bewusst, dass sie einen wertvollen und wichtigen sozialen Auftrag ausführte. Durch Erfahrungen, Reflexion und Wissen bildete sie ihr Selbstverständnis aus und entwickelte sich immer mehr zu einem präventiv sozialarbeiterischen Angebot. Die SIP versteht sich heute

\footnotetext{
${ }^{1}$ Sozialarbeiterin FH, Bereichsleiterin SIP Stadt Luzern.

2 Soziokulturelle Animatorin in Ausbildung, Mitarbeiterin SIP Stadt Luzern.

${ }^{3}$ Vgl. www.stadtluzern.ch/sip. Aus Gründen der Lesbarkeit wird im folgenden Text SIP geschrieben, wobei immer die SIP der Stadt Luzern gemeint ist.
} 
als professionelle Akteurin der aufsuchenden und vermittelnden Sozialarbeit für eine breite Zielgruppe.

Den Autorinnen ist es wichtig, diese Entwicklung aufzuzeigen. Einerseits sind sich wenige PolitikerInnen bewusst, wie wertvoll die SIP-Arbeit für eine Stadt sein kann. Anderseits sehen Fachkräfte oft nicht, dass gerade in diesem Spannungsfeld zwischen Beziehungsarbeit und Ordnungsdienst ein wichtiger sozialer Auftrag steckt, möglicherweise eine neue Spezialisierung der Sozialen Arbeit. Denn bei Differenzen vermitteln und nachhaltig einwirken zu können und soweit als möglich mit den Konfliktparteien Lösungen $\mathrm{zu}$ suchen und zu bestimmen, setzt eine qualifizierte und vielseitige Ausbildung in der Sozialen Arbeit, insbesondere im Konfliktmanagement voraus.

\section{Geschichte, Auftrag und Arbeitsverständnis der SIP}

\subsection{Geschichte}

Wie in anderen Städten gab es in den 1990er-Jahren auch in Luzern eine offene Drogenszene und zunehmende Schwierigkeiten mit übermässigem Alkoholkonsum im öffentlichen Raum. Der Verein Kirchliche Gassenarbeit betrieb einen Spritzenbus, ein «Fixerstübli» und aufsuchende Gassenarbeit, mitfinanziert durch die Stadt Luzern. 2005 beschloss der Stadtrat, nebst bestehenden repressiven Massnahmen einen sozialarbeiterischen Ansatz einzuführen, um die Problematik anzugehen.

Als Vorbild diente das Konzept der sip züri. In Zusammenarbeit mit der Hochschule für Soziale Arbeit Luzern erarbeitete die Stadt Luzern ein Methodenhandbuch für eine SIP. Als Ansätze wurden der präventive Ordnungsdienst und die Soziale Arbeit aufgeführt. Bemerkenswert ist, dass die Soziale Arbeit darin als «Uniformierte Sozialarbeit» und «Vermittelnde Sozialarbeit» festgehalten ist (Stadt Luzern 2009: 7-8).

Im Jahr 2008 anerkannte das Stadtparlament die SIP als neuen Fachbereich. Im Jahr 2015 wurde im Rahmen eines Sparpakets über die Auflösung der SIP diskutiert, was aber vom Stadtrat abgelehnt wurde. Der Entscheid stützte sich auf den Projektbericht Haushalt im Gleichgewicht (Stadt Luzern 2014: 1), in dem empfohlen wird: «Eine ersatzlose Aufhebung der 
SIP ist aufgrund der Analyse strikte abzulehnen. Die Analyse zeigte, dass die Einsparungen bei einer Aufhebung gegenüber möglichen Folgekosten in unbekannter Höhe, dem bei weitem entgegenstehe». Seither ist die Auflösung der SIP kein Thema mehr.

Per 1. Januar 2018 wechselte die SIP von der Umwelt- und Verkehrsdirektion in die neu gebildete Dienstabteilung Quartiere und Integration (QUIN) der Sozial- und Sicherheitsdirektion der Stadt Luzern. Die QUIN vereint die Quartierarbeit, die Quartierentwicklung, die Fachstelle Integration und die SIP. Die Reorganisation zeigt, dass die SIP mit ihren Aufgaben die Quartierarbeit und die Fachstelle Integration stärkt und für ein soziales Zusammenleben und eine gelungene Integration gewichtige Arbeit leistet.

\subsection{Auftrag}

Der Kernauftrag der SIP ist die Soziale Sicherheit und deren Regulierung im öffentlichen Raum, mit dem Ziel einer respektvollen Koexistenz aller Personen und Personengruppen, die sich im öffentlichen Raum aufhalten, insbesondere Jugendliche und randständige Menschen. Die SIP bearbeitet Konfliktfelder, die aufgrund von Verhaltensnormen und gesetzlichen Regelungen entstehen. Es handelt sich dabei insbesondere um zwischenmenschliche Konflikte und Nutzungskonflikte. Die SIP agiert in einer Kombination von helfender, aufsuchender und vermittelnder Sozialarbeit und ordnungsdienstlicher Tätigkeit.

Wie im Leitbild der SIP definiert, ${ }^{4}$ schliesst sie die Lücke zwischen Sozialer Arbeit und Polizei. Sie trägt durch ihre Präsenz und durch ihre gezielten Interventionen dazu bei, das Sicherheitsgefühl der Personen im öffentlichen Raum zu erhöhen, allen Anspruchsgruppen einen öffentlichen Aufenthaltsraum in der Stadt zu gewährleisten und damit die Lebensqualität in der Stadt Luzern zu erhalten. Gleichermassen ist sie mandatiert, niederschwellige Soziale Arbeit für Personen zu leisten, die kaum institutionelle soziale Hilfe in Anspruch nehmen.

Menschen mit einer Sucht halten sich «gerne» und oft im öffentlichen Raum auf. Da ihre finanziellen Mittel limitiert sind und ihr früheres Beziehungsnetz oft nicht mehr existiert, werden Strassen und Plätze zu ihrem

\footnotetext{
${ }^{4}$ Vgl. SIP-Leitbild der Stadt Zürich: www.stadtluzern.ch/thema/3943, Zugriff 06.02.20.
} 
neuen Lebens- und Begegnungsraum, in welchem sie KollegInnen treffen und Freundschaften knüpfen. Die Aussenräume werden zu ihrer «Kneipe», in der sie günstig ihr Bier trinken können. Der Stadtraum wird zu ihrem kostenlosen Schlafplatz und zu ihrem Informationsort, wo sie erfahren, wer sich wo aufhält und wo Hilfe zu erwarten ist. Bestimmte Plätze und Quartiere werden zum «Marktplatz», auf dem sie Drogen kaufen können. In belebten Parkanlagen und Plätzen erleben sie, dass auch sie der Gesellschaft angehören. Die SIP, die täglich im öffentlichen Raum unterwegs ist, wird wie die Gassenarbeit zu einer sehr niederschwelligen Anlaufstelle der Sozialen Arbeit.

Durch Gespräche und Beobachtungen zeigt sich einerseits, dass genau diese Menschen der Unterstützung der SIP bedürfen. Anderseits sollen Kinder, Jugendliche, erwachsene Menschen und ältere Personen, die sich bei einer übermässigen Ansammlung von suchtbetroffenen Menschen unsicher fühlen könnten, Schutz erhalten. Diesem Dilemma begegnet die SIP mit Präsenz, Gesprächen und Massnahmen, die weder die eine noch die andere Partei voll und gänzlich ausgegrenzt und die schwächere Partei stützt. Ein treffendes Beispiel ist der zentrale Park und Spielplatz «Vögeligärtli». Hier stellt sich die «Spagataufgabe», die Suchtbetroffenen dafür zu sensibilisieren, das Konsumieren vor Ort zu unterlassen oder aber den Park zu verlassen und für den Suchtmittelkonsum die K\&A aufzusuchen, damit die Kinder unbeschwert und sicher den Spielplatz nutzen können. Letztendlich sollten nicht politische Gründe, sondern Sachlage, soziales Denken und «gesunder Menschenverstand» die Interventionen der SIP leiten.

\subsection{Umsetzung}

Die Erfahrung zeigt, dass eine Verbesserung der Lebenslage oder ein Ausstieg aus der Sucht möglich sind, wenn suchtbetroffene Menschen fähig werden, selbst ihren Alltag mit kleinsten Veränderungen neu zu gestalten. Dies gelingt, wenn kontinuierliche Beziehungsarbeit mit den KlientInnen geleistet wird. Durch die wiederholten Kontaktaufnahmen und Gespräche fassen sie Vertrauen und zeigen sich eher bereit, selbstverantwortlich zu handeln. 
Die SIP sieht sich mitverantwortlich, Menschen den Zugang zur Gesellschaft und zu den vielfältigen Angeboten, welche die Stadt und die Sozialnetze bereitstellen, zu ermöglichen. Wenn eine Klientin im Gespräch den Wunsch nach einer Arbeitstätigkeit äussert, informieren die SIP-Mitarbeitenden über mögliche Angebote, beispielsweise über den Verein «Jobdach». Dieses Angebot ermöglicht Menschen mit erschwerten Bedingungen, einer Beschäftigung nachzugehen, und bietet ihnen gleichzeitig eine Tagesstruktur. Entweder geben die SIP-Mitarbeitenden der Person eine Visitenkarte ab oder begleiten sie zum gewünschten Ort.

Weiter interveniert die SIP bei Drogen- oder übermässigem Alkoholkonsum und engagiert sich stark in der Sucht- und Gewaltprävention. Sie versucht, frühzeitig die Auswirkungen einer Sucht anzusprechen, und deeskaliert bei Konfliktsituationen, um Strafvorfälle und damit unnötige Strafmassnahmen zu verhindern. Ist erkennbar, dass sich ein Konflikt ausweitet oder Personen gefährdet sind, zieht die SIP mit Vorankündigung die Polizei hinzu.

Als Akteurin im städtischen Sozialraum ist sie an jedem Wochentag bis spät in die Nacht für alle Personen und insbesondere für randständige Menschen da. Dadurch schliesst sich eine Lücke, wenn wichtige Leistungserbringende, wie die Kontakt- und Anlaufstelle der Gassenküche, die Gassenarbeit oder weitere wichtige Institutionen in den späten Abendstunden nicht mehr verfügbar sind.

\subsection{Arbeitsverständnis und Methoden}

Die Professionellen der Sozialen Arbeit stützen ihr Handeln auf Grundwerte der Menschenwürde und der Menschenrechte. Demnach stehen jedem Menschen, ungeachtet von Geschlecht, Hautfarbe, Ethnie, Status und Individualität, die Achtung und der Schutz seiner Menschenwürde sowie das Recht auf Gleichwertigkeit zu (vgl. Schmocker 2010: 8). Als soziale Organisation bezieht sich die SIP auf diese Wertehaltung und auf ein humanistisches Menschenbild. Dieses besagt, dass der Mensch grundsätzlich gut ist, dass er nach einem selbstbestimmten und sinnerfüllten Leben strebt und dazu soziale Kontakte sucht. Im Methodenhandbuch der SIP ist diese Grundhaltung verankert. Es hält beispielsweise Folgendes zum Thema Empowerment fest: «Die strategische Idee des Empowerment ist es, 
die Anspruchstellenden in die Verantwortung zu nehmen, in ihren Wahrnehmungs- und Handlungsfähigkeiten $\mathrm{zu}$ fordern und $\mathrm{zu}$ fördern und ihnen damit die Kraft (Power) zugänglich zu machen, die sie selbst aufgrund ihrer Ressourcen und Potenziale mitbringen» (Stadt Luzern 2009: 16).

Die Arbeitsweise der SIP basiert auf Theorien und Modellen wie der Kommunikationstheorie nach Watzlawick (vgl. 2011) und dem Kommunikationsmodell nach Schulz von Thun (vgl. 1981), der Klientenzentrierten Gesprächsführung nach Rogers (vgl. 2017), der Gewaltfreien Kommunikation nach Rosenberg (vgl. 2013), der Motivierenden Gesprächsführung (Motivational Interviewing) nach Miller und Rollnick (vgl. 2015), den Konfliktlösungsansätzen nach Scharmer (vgl. 2013), dem Ansatz des Empowerment nach Herriger (vgl. 2014) und der Lebensweltorientierung nach Thiersch (vgl. 2005).

Parallel dazu wird im Methodenhandbuch für den Ordnungsdienst die Thematik der Repression behandelt (Stadt Luzern 2009: 11): «Repression im Sinne der Anwendung von Staatsgewalt oder Macht, auch gegen den Willen einer anderen Partei. Es ist zu beachten, dass die SIP keine physische Gewalt gegen KlientInnen anwendet, sondern mit «weichen Methoden» reguliert, da sie über keine polizeihoheitlichen Kompetenzen verfügt». Das bedeutet, dass die Verantwortung, ob Repression angewendet wird, der Klientel übergeben wird. Der Beizug der Polizei wird angekündigt, sodass die KlientInnen die Möglichkeit haben, entsprechend zu reagieren. Dadurch entsteht ein transparentes und interaktives Gespräch, indem Problemlösungen gemeinsam erarbeitet werden.

Die Auswertungen der erfolgreichen Interventionen zeigen, dass nicht nur das Handwerk oder das Werkzeug ausschlaggebend sind - vielmehr ist es eine Frage des grundsätzlichen Umgangs mit der Situation und mit den betroffenen Menschen sowie der Art und Weise, wie die Werkzeuge eingesetzt werden. Das Methodenhandbuch der SIP Luzern verweist unter Interventionsgrundsätze auf Folgendes: «Ob der Zugang zu einem Menschen (in einem beliebigen Bewusstseinszustand) hergestellt werden kann, ist in erster Linie eine Frage der inneren Haltung diesem Menschen gegenüber. Jeder Mensch spürt intuitiv, ob die Person, die ihn 
anspricht, dies aus einer wohlwollenden, offenen oder aus einer abschätzenden, geschlossenen Haltung ihm gegenüber tut» (Stadt Luzern 2009: 17).

Eine weitere wichtige Komponente des Interventionsverständnisses und der inneren Haltung ist es, sich vor Augen zu halten, dass jedes Verhalten (und sei es noch so seltsam, unangenehm und unverständlich) seinen Grund hat und oft sogar eine Überlebensstrategie bedeutet (Stadt Luzern 2009: 15).

Weiter ist das «Pacing» (Stadt Luzern 2009: 19) zu erwähnen: «Wenn es zu einer Kontaktaufnahme kommt, besteht der erste Schritt darin, die Wellenlänge zu suchen, auf der das Gegenüber sendet. Nachher gilt es, sich auf diese Wellenlänge einzustellen». Es ist wichtig, den Kommunikationsverlauf zu beobachten: Blockiert, öffnet, bereichert die Person die Interaktion? Dazu kann es hilfreich sein, während des Gesprächs auf Augenhöhe niederzuknien.

Die SIP ist bemüht, ihrer Klientel neue Handlungsfelder und Beziehungen zu erschliessen. Um solche Prozesse anzustossen, braucht es Zeit und regelmässige persönliche Begegnungen. Die SIP investiert in beide Komponenten. Bei der KlientInnenarbeit, so Gahleitner (2017: 275), «ist zu berücksichtigen, dass nicht nur gezielte Beratungs- und Therapiesitzungen, sondern die alltägliche Kommunikation über Banalitäten eine wichtige verbindende und stabilisierende Rolle spielt».

Bei Konflikten ist die SIP die gefragte oder ungefragte Vermittlerin. Streiten sich zwei oder mehrere Parteien, spricht die SIP die KonfliktträgerInnen an und bietet Hilfestellung zu Lösungen. In ihrer Funktion ergreift sie Partei für den öffentlichen Raum und die Rahmenbedingungen für eine stadtverträgliche Koexistenz. Ansonsten ist die SIP allparteilich. Sie bevorteilt weder die eine noch die andere Partei. Herausfordernd wird es, wenn die Interessen sehr divergent sind. Zum Beispiel, wenn ein Konzertveranstalter die Meinung vertritt, dass sich in der Nähe der Aufführung keine Menschen mit einem Suchtproblem aufhalten dürfen, oder wenn die Ansicht besteht, dass der Bahnhofplatz kein Treffpunkt für suchtbetroffene Menschen sein darf.

Die SIP-Mitarbeitenden sind wichtige BotschaftsträgerInnen. Sie leiten täglich Sachverhalte weiter, seien es Mitteilungen von BürgerInnen, 
von der Behörde oder aus der Gesetzgebung. Idealerweise wird ein direkter Kontakt hergestellt. Ist dies nicht möglich, bringt die SIP die Position der suchtbetroffenen Menschen ein. Wenn BürgerInnen zum Beispiel verlangen, dass Randständige auf einem Platz wie dem Europaplatz vor dem Bahnhof nicht zu tolerieren seien, weisen wir darauf hin, dass der öffentliche Grund für alle Gruppierungen zugänglich ist, sofern sie sich an die allgemeinen Regeln halten.

Wie diese Beispiele zeigen, nimmt die SIP die Haltung der Vermittlerin ein, was die Bezeichnung «vermittelnde Sozialarbeit» bestätigt.

\subsection{Stellenwert der Sozialen Arbeit}

In der professionellen Suchthilfe ist ein fundiertes Fachwissen über die Suchtproblematik und deren weiteren Zusammenhänge unabdingbar. Für die Interventionen sind Kompetenzen und Methoden in Gesprächsführung, Konfliktlösung, Beratung und Systemwissen notwendig. Ferner ist in der niederschwelligen Sozialen Arbeit im öffentlichen Raum Fachwissen zu den Themen Jugend und Alter, Gesundheit, Soziale Sicherheit, Integration, Migration, Recht usw. gefragt. Alle diese Kenntnisse und Fähigkeiten sind für ein professionelles Handeln bei der Arbeit der SIP zentral. Deshalb ist es von grossem Vorteil, wenn die Mitarbeitenden eine Ausbildung im Bereich der Sozialen Arbeit oder in verwandten Fachbereichen ausweisen können. Dies ermöglicht ihnen zudem den Besuch von Weiterbildungsangeboten an den Fachhochschulen. Besteht keine tertiäre Ausbildung, ist die Teilnahme an fachspezifischen Weiterbildungen, wie zum Beispiel zu Sucht- oder Gewaltthemen, eingeschränkt.

Die SIP hält ihre Beobachtungen und Erkenntnisse systematisch fest. Erhebungen zu Zu- oder Abnahme der Drogenszene in einem Stadtteil, der Gesundheitszustand von älteren, suchtbetroffenen Menschen, welche Plätze von wem und wie stark frequentieret werden, ob ein friedliches Miteinander an einem Brennpunkt möglich ist: All diese Informationen leitet die SIP an die Behörde und an Fachgremien weiter. Dabei wird der Persönlichkeitsschutz der betroffenen Personen stets vollumfänglich gewahrt.

Die Behörden und Institutionen nutzen diese Beobachtungen und Erkenntnisse für strategische Entscheidungen. Folgendes Beispiel veranschaulicht dies: Auf einem zentralen, bei Familien und Kindern beliebten 
Stadtpark droht eine Szenenbildung. Die SIP stellt sich folgende Fragen, die sie bei den relevanten Stellen einbringt: Verträgt sich die Zunahme von Suchtbetroffenen auf dem öffentlichen Platz mit den Kindern, die sich dort aufhalten? Fehlt suchtbetroffenen Personen ein geschützter Ort, an dem sie sich aufhalten können? Zieht der Aufenthalt der Suchtbetroffenen die Zunahme von Drogenhandel nach sich? Werden die öffentlichen Toiletten nach dem Konsum sauber zurückgelassen und bleiben sie für weitere BenutzerInnen zugänglich? Solche Überlegungen bewirken, dass auf öffentlichem Grund überlegte regulierende Aktivitäten entstehen oder dass bauliche Massnahmen getroffen werden.

Nebst den städtischen Stellen nutzen auch kantonale und ausserkantonale Fachstellen die Beobachtungen, das Wissen und die Erfahrung aus der Sicht der Sozialen Arbeit. In der Fachgruppe «Schadensminderung» 5 des Kantons Luzern, dessen Auftrag es ist, Trends zu analysieren und die Angebote der Suchthilfe zu überprüfen, wies die SIP auf die zunehmende Verschlechterung des Gesundheitszustandes von alternden suchtbetroffenen Personen auf der Strasse hin. Mit diesem Hinweis verbalisierte die SIP eine Zukunftsproblematik, der sich auch der Kanton Luzern stellen muss.

Ein weiteres Beispiel ist die Vernetzung mit «Safer Clubbing», einem Zusammenschluss von Clubs und Nachtlokalen in Luzern. Diese laden jeweils die Fachstelle Akzent Prävention und Suchthilfe, die Polizei, den Sicherheitsbeauftragen der Stadt Luzern und die SIP halbjährlich zu ihrem «runden Tisch» ein. Das Ziel dieser Arbeitsgruppe ist die Förderung der Suchtprävention und die Sicherheit der Menschen im Ausgang.

Nachhaltige Lösungen bei regulativen Aufgaben erforden eine umfassende Sichtweise. Die Soziale Arbeit ist in solchen gesellschaftlichen Herausforderungen eine kompetente Disziplin, weil sie sich gemäss ihrer Definition sowohl auf Theorien menschlichen Verhaltens als auch auf The-

\footnotetext{
${ }^{5}$ Die kantonale Fachgruppe «Schadensminderung» (KFG) setzt sich zusammen aus VertreterInnen der folgenden Akteure: Luzerner Polizei, Drop-in der Luzerner Psychiatrie, Verein Kirchliche Gassenarbeit, Jobdach, IG Arbeit, ZISG Zweckverband für institutionelle Sozialhilfe und Gesundheitsförderung, Kantonales Gesundheits- und Sozialdepartement, Städtische Soziale Dienste und SIP.
} 
orien sozialer Systeme stützt. Sie ist Vermittlerin an Orten, an denen Menschen und ihre sozialen Umfelder aufeinander einwirken (Schmocker 2010: 8).

\section{Interdisziplinäre Zusammenarbeit und angrenzende Arbeiten}

Die SIP pflegt die interdisziplinäre Zusammenarbeit. Sie ist seit der Eingliederung in die Dienstabteilung QUIN Teil des neuen Kompetenzzentrums rund um die Sozialintegration in den städtischen Quartieren. Auch die Leitung der SIP nimmt regelmässig an «Runden Tischen» teil. Exemplarisch zeigt sich dies am Beispiel der Fachgruppe Schadensminderung. Die SIP wurde 2017 angefgragt, ob sie in diesem Gremium mitwirken wolle. Dort vertritt sie den Ordnungsdienst und den Aspekt der aufsuchenden Sozialen Arbeit.

Weitere wichtige PartnerInnen sind die Polizei, die Kindes- und Erwachsenenschutzbehörde KESB, die Beistandschaften, die Sozialdienste, die kirchliche Gassenarbeit usw., mit denen jeweils die Zuständigkeiten geregelt sind.

Da die SIP weder Fallbearbeitung noch Langzeitbetreuung anbietet, hat sie sich in der Triage zu spezialisieren. Bei freiwilligen Erstgesprächen wird eruiert, ob die KlientInnen gewillt sind, Hilfe anzunehmen, von wem und wie ihr persönlicher Beitrag aussieht. In Ausnahmefällen organisiert die SIP eine HelferInnen-Konferenz. Wenn nötig und erwünscht, begleiten die SIP-Mitarbeitenden beim ersten Mal die KlientInnen an die gewünschte Stelle wie Arztpraxis, KESB, psychiatrische Klinik, auf den Zug, ans Gericht, in den Strafvollzug etc. Die Freiwilligkeit und die Eigenverantwortung der KientInnen sind jedoch immer die Grundlage für den folgenden Schritt.

Die SIP interveniert bei Selbst- und Fremdgefährdung. Äussern KlientInnen mehrmals suizidale Gedanken, werden wir aktiv. Für diese Aufgabe ist es notwendig, breit vernetzt zu sein und über fundiertes sozialarbeiterisches und psychologisches Wissen zu verfügen. $\mathrm{Zu}$ erwähnen ist, dass die SIP nie Zwangsmassnahmen durchführt.

Eine intensive Zusammenarbeit pflegt die SIP mit der Polizei. Die Kompetenzen beider Institutionen sind zwar sehr unterschiedlich, doch 
das beiderseitige Wissen und die jeweiligen Erfahrungen ermöglichen die gegenseitige Unterstützung. Zweimal im Monat findet ein Austausch zwischen der Polizei und der SIP statt. Er dient dazu, sich in punkto Aufgabenerfüllung abzusprechen. Es wird bestimmt, welche der beiden Organisationen wie und wann an den Brennpunkten betreffend Suchtproblematiken Präsenz zeigt und Verantwortung übernimmmt. Der Datenschutz wird stets gewährleistet. Die SIP arbeitet nicht für die Polizei und ist verschwiegen. Dies ist äusserst wichtig in der Beziehungsarbeit mit den KlientenInnen, um das Vertrauensverhältnis aufrechtzuerhalten.

Interdisziplinäres Zusammenarbeiten findet auch innerhalb der SIP statt. Im Team arbeiten Fachleute aus den Fachrichtungen Soziale Arbeit, Sozialpädagogik und soziokulturelle Animation und aus angrenzenden Berufen des Gesundheits- und Asylwesens, der Schule und des kaufmännischen Bereichs. Jedes Teammitglied bereichert mit seinem Spezialwissen die Arbeit der SIP . Fachdiskurse ergeben sich bei der Fallbearbeitung, an Teamsitzungen, an Super- und Intervisionen. Bei spezifischen Sachfragen bearbeitet der oder die entsprechende SpezialistIn das Thema. Zum Beispiel: Um ein Statement dazu abzugeben, ob in Luzern Cannabis reguliert werden soll, holt die Leitung auch das Fachwissen des Mitarbeiters ein, der die Fachausbildung Peer im Bereich Sucht aufweist.

\section{Stand der Professionalisierung der Sozialen Arbeit bei der SIP}

Wie schon erwähnt, ist das strategische Ziel der SIP Luzern, die Koexistenz aller Bevölkerungsgruppen zu erwirken und zu erhalten. Mit diesem Arbeitsziel nimmt die SIP durch die tägliche Präsenz im öffentlichen Raum Aufgaben der Sozialen Arbeit wahr, um die Beteiligung am Gesellschaftsleben zu fördern, Hilfeleistungen zu erbringen, ein Miteinander und Nebeneinander zu ermöglichen oder Bedürfnisse zu vertreten. Für alle SIPMitarbeitenden ist es zentral, unter dem Verständnis der Profession Soziale Arbeit diese Koexistenz und Beteiligung aller beteiligten Nutzungsgruppen im öffentlichen Raum wirkungsvoll zu fördern, wie es auch das Methodenhandbuch vorsieht (Stadt Luzern 2009: 6). Die SIP-Mitarbeitenden verfügen für den präventiv ordnungsdienstlichen Auftrag keine poli- 
zeilichen Kompetenzen. Damit bewegt sich die Arbeit in einem Graubereich und ist näher der Sozialen Arbeit zuzuordnen. Ein Mitarbeiter meint: «Die SIP setzt dort an, wo die Polizei aufgrund von Zeitdruck und Gesetz zu wenig auf das Anliegen der Klientel eingehen kann. Diese Lücke schliessen wir durch Anteilnahme und Empathie, mit welchen wir auf Menschen zugehen und deren Bedürfnisse abholen». Eine weitere Mitarbeiterin äussert: «Regulierend arbeiten ohne Repression ist nicht immer einfach, jedoch ein Credo für die SIP Luzern. Wir haben erkannt, dass Anteilnahme, Anerkennung, Achtung und Respekt, alles Fähigkeiten der Empathie, nachhaltig unseren Auftrag unterstützen».

Das Verständnis, dass die SIP niederschwellige Soziale Arbeit leistet, ist bei der Verwaltung, bei den Kunden, bei den Institutionen und bei der Bevölkerung gewachsen. Es wird anerkannt, dass die Aufgaben vielseitig und komplex sind und bildlich gesprochen ein «Puzzle» darstellen, welches mit Wissen aus den Bereichen Soziales, Gesundheit, Recht und Ethik immer wieder neu reflektiert und bearbeitet werden muss.

In der Prävention tätig zu sein, setzt eine ständige Auseinandersetzung zu Fragen der Wertung, Normativität, Moral und Ethik voraus. Es ist falsch anzunehmen, dass präventive Massnahmen a priori gewinnbringend sind. Es ist stets zu prüfen, ob die Prävention langfristig die Risiken eindämmt oder ob sie sich eher negativ auswirkt. Es ist ungewiss, ob das Weglassen der Prävention die bestehende Gefahr erhöht oder ob genau dies zur Genesung führt. Martin Hafen (vgl. 2013) plädiert dafür, die Werte und Bewertungen der Prävention immer wieder kritisch zu hinterfragen. Er hält die Fachleute und Organisationen in Prävention und Gesundheitsförderung an, Strukturen einzurichten, die es erlauben, möglichst partizipativ relevante Fragen aus der Perspektive der Präventionsethik zu diskutieren. Für die SIP ist es unumgänglich, nebst der Arbeit auf der Strasse diese Diskussion zu führen.

Die Professionalisierung in der aufsuchenden und vermittelnden Sozialarbeit hat Entwicklungspotential. Da nur wenige Städte eine solche Institution finanzieren und die SIP-Arbeit als solche erst seit wenigen Jahren existiert, gibt es keine spezifische Ausbildung dazu. Bei den Fach(hoch)schulen in der Schweiz werden weder Module noch spezifische Lehrgänge angeboten. Forschungsvorhaben gibt es unseres Wissens erst 
seit kurzem und fachbezogene, wissenschaftliche Arbeiten oder Expertisen sind uns keine bekannt. Die SIP evaluierte einzig die Wertschöpfung mit ihren Daten, jedoch nicht den Methodenansatz.

Gerade, weil das Arbeitsfeld der SIP so neu ist, braucht es eine Professionalisierung. Dieser Prozess wird in erster Linie durch Erfahrungen und deren Reflexion vorangetrieben. Extern holt die SIP das Wissen und die Kompetenzen aus der allgemeinen Sozialen Arbeit oder den angrenzenden Berufsfeldern. Die Mitarbeitenden bilden sich an verschiedenen Fachtagungen weiter. Die oben erwähnten fehlenden Ausbildungsmöglichkeiten an den Fach(hoch)schulen verhindern eine anerkannte Professionalisierung. Als Anbieterin von Ausbildungsplätzen/Praktikumsplätzen für AbsolventInnen der Fach(hoch)schulen ist die SIP immer noch sehr umstritten.

Es existiert wenig Austausch und Vernetzung zwischen den Städten und Gemeinden, die ein SIP-Angebot implementiert haben. ${ }^{6}$ Die strategischen Ausrichtungen der Institutionen variieren. In einigen Städten liegt der Fokus der SIP-Arbeit eher auf dem Aspekt der Sicherheit, in anderen auf dem der Sozialen Arbeit. An Tagungen mit sozialen Themen sind die SIP-Angebote kaum vertreten. Zudem gibt es noch keine spezifische Fachliteratur. Dies alles lässt erahnen, dass das Berufsfeld der SIP aktuell in der Sozialen Arbeit ungenügend verankert ist und somit wenig vom Knowhow anderer Organisationen profitiert.

Zusammenfassend kann gesagt werden: Beide Seiten, die Profession der Sozialen Arbeit und die Organisationen der aufsuchenden und vermittelnden Sozialarbeit, haben für die Anerkennung des Arbeitsfeldes und deren Konsolidierung in der Sozialen Arbeit noch «Hausaufgaben» wie die Organisation von Fachtagungen oder eine bessere nationale Vernetzung zu erledigen. Dazu müssten jedoch auch die nötigen Ressourcen gesprochen werden.

\footnotetext{
6 Ähnliche Organisationen wie die SIP bestehen in Zürich, Bern, Biel, Genf, Langenthal, Lausanne, Basel, Kanton Uri, Luzern u. a.
} 


\section{Strukturelle Aspekte und Rahmenbedingungen}

Es existieren keine gesetzlichen Vorgaben für die Gemeinden, eine Institution wie die SIP zu implementieren. Die Verwaltung der Stadt Luzern mit einer SIP zu erweitern, war ein politischer und ein parlamentarischer Beschluss. Um eine professionelle und nachhaltige Umsetzung zu gewährleisten, ist es notwendig, klare Leitsätze mit entsprechenden Vorgehensweisen zu definieren. Die Unterstützung durch die Hochschule für Soziale Arbeit Luzern war hier sehr wertvoll. Das Methodenhandbuch ist noch heute in den meisten Belangen eine wichtige Grundlage für die tägliche Arbeit. Es enthält Kapitel zu den Arbeitsfeldern, den Interventionsstrategien und -grundsätzen, zur Teamarbeit, zum Umgang mit KlientInnengruppen, zu speziellen Werkzeugen (Skills), zur Vernetzung und auch zum politischen Ansatz (Stadt Luzern 2009: 6). Eine regelmässige Aktualisierung ist jedoch erforderlich.

Im Laufe der Zeit wurde der Stellenetat erhöht und danach wieder reduziert. Heute sind es bei der SIP Luzern 550 Stellenprozente. Zusätzlich unterstützen ein bis zwei Zivildienstleistende jährlich das Team.

Der prozentuale Anteil an ausgebildeten Sozialarbeitenden ist im Stellenplan festgehalten: 290 Stellenprozente mit Abschluss in Sozialer Arbeit und 260 Stellenprozente mit Abschlüssen in verwandten Berufen. ${ }^{7}$

Für die vielfälltigen Aufgaben setzt sich das Team interdisziplinär zusammen und ergänzt sich fachlich.

Die Ausgaben der SIP setzen sich zusammen aus 90\% Lohnkosten und 10\% für Aus- und Weiterbildungen, Arbeits- und Verbrauchsmaterial, Arbeitskleider sowie Kosten für die eigene Software.

Die SIP ist täglich an allen Werk-, Sonn- und Feiertagen unterwegs. Die Präsenzzeiten variieren je nach Jahreszeiten und Wochentagen. Die Mitarbeitenden sind bis 24:00 Uhr unterwegs. ${ }^{8}$ Eine längere Nachtpräsenz ist nicht gewinnbringend, da durch zu hohen Alkoholkonsum ausgelöste Problemfelder letztendlich mit polizeihoheitlichen Kompetenzen gelöst werden müssen. Diese stehen jedoch den SIP-Mitarbeitenden nicht zu.

\footnotetext{
7 Im Team arbeiten Fachleute aus den Fachrichtungen Sozialarbeit, Sozialpädagogik, soziokulturelle Animation und aus angrenzenden Bereichen wie Sozialwissenschaften, Migration, Sozialbegleitung.

${ }^{8}$ Dienst 1 bis um 20:15 Uhr, Dienst 2 bis um 22:00 Uhr und Dienst 3 bis um 24:00 Uhr.
} 
Die SIP ist halbuniformiert. Gründe dafür sind die gute Erkennbarkeit durch die Bevölkerung, die so erkennt, in wessen Auftrag die Mitarbeitenden unterwegs sind. Es wurde darauf geachtet, dass sich die Bekleidung von der Uniform der Polizei unterscheidet.

Der bereits im Kapitel 2 erwähnte Abteilungswechsel (Strukturwechsel) von «Stadtraum und Veranstaltungen» zu QUIN veränderte das Aufgabenfeld der SIP. Vorher standen Kontrollaufgaben bei Veranstaltungen und der Nutzung von öffentlichen Flächen stärker im Fokus. Heute arbeitet die SIP vermehrt sozialarbeiterisch und sozialräumlich. Ihr Engagement in der Suchtpolitik ist breiter; hauptsächlich in den Bereichen der Prävention, Gesundheitsförderung und Jugendschutz.

\section{Herausforderungen und Ausblick}

\subsection{Herausforderungen}

Die Veränderungen im öffentlichen Raum sind in der Arbeit mit Suchtbetroffenen eine der Herausforderungen für die SIP Luzern. Verändert hat sich, dass der öffentliche Raum in den letzten Jahren immer stärker als Treff- und Aufenthaltsraum verschiedenster Menschengruppen genutzt wird. Brachen, in denen sich Randgruppen ungestört aufhalten können, werden immer seltener. Der Handel mit Drogen wird öffentlich hemmungsloser getätigt. Verkaufsstellen von Alkohol haben zugenommen und bieten längere Öffnungszeiten an. Der Ausbau des öffentlichen Verkehrs bringt mehr Menschen am Abend in die Stadt. Die Zunahme von Konzertanlässen und Events erfordern mehr Präsenz und schmälern die Suchtpräventionsarbeit. Der liberalere Umgang mit Suchtmitteln birgt ein Spannungsfeld, das bei der Betreuung und Begleitung von Suchtbetroffenen nicht zu unterschätzen ist. Klare Absprachen mit den AkteurInnen im Suchtbereich sind umso wichtiger. Alle diese Veränderungen erhöhen das Konfliktpotential und reduzieren bei der SIP die wichtige Beziehungsarbeit.

Besonders die Präventions-, und Sicherheitsfragen auf der Mikro-, Meso- oder Makroebene für Suchtbetroffene und Jugendliche würden mehr Ressourcen benötigen. Eine Erweiterung von Stellenprozenten ist politisch schwierig. Nur schon die Bestehenden zu erhalten bedarf eines 
ständigen Ringens bei der Finanzierung, besonders bei Sparrunden. Da die SIP nicht gesetzlich verankert ist, sondern einer parlamentarischen Massnahme unterliegt, sind ihr Weiterbestand und ihre Ausrichtung immer wieder Teil der politischen Aushandlung.

Eine weitere Herausforderung ist, dass in der Nacht und an den Wochenenden wichtige PartnerInnen für Hilfeleistungen wie Ärztepersonal für eine Klinikeinweisung oder eine Wohnheimleitung für eine Übernachtungsmöglichkeit schwer abrufbar sind. Auch die bestehende Notschlafstelle ist nur begrenzt offen und schliesst gewisse Personengruppen aus.

\subsection{Ausblick}

Als Folge des gesellschaftlichen Wandels und der damit einhergehenden Anforderungen muss der Grundauftrag mit den Zielen der SIP immer wieder neu reflektiert und nach den Erkenntnissen angepasst umgesetzt werden. Erfolgreich ist die Arbeit dann, wenn das Handeln zielgerichtet bleibt, sich auf die Ansätze der Sozialen Arbeit als Handlungswissenschaft im Lichte der Definiten der International Federation of Social Workers $(\text { IFSW })^{9}$ abstützt und mit den Leitsätzen der Stadt vereinbar ist.

Es ist ein Gewinn, dass die SIP heute der Direktion Soziales und Sicherheit unterstellt ist. Durch diesen Strukturwechsel sind die beiden Disziplinen näher zueinander gerückt. Das Verständnis, Sicherheitsfragen mit Ansätzen und Methoden der Sozialen Arbeit zu bewältigen, hat sich merklich erhöht.

Das Bedürfnis grösserer Gemeinden, eine Institution wie die SIP aufzubauen, wächst. Doch eine Umsetzung scheitert oft auf der politischen Ebene. Mögliche Gründe sind: Die Wertschöpfung einer solchen Institution ist zu wenig bekannt, belegt und garantiert, Know-how, finanzielle Mittel und die politische Attraktivität, Sicherheitsprobleme mit dem oben erwähnten Arbeitsansatz anzugehen, fehlen.

Die Autorinnen äussern die Behauptung: Würden tertiäre Ausbildungsstätten das Arbeitsfeld «Aufsuchende und vermittelnde Sozialar-

\footnotetext{
${ }_{9}^{9}$ Globale Definition der Profession Soziale Arbeit der IFSW: www.tinyurl.com/yyjqo9z4, Zugriff 06.02.2020.
} 
beit» in die Ausbildung integrieren und als eigene Fachrichtung anerkennen, würde dieses neue Berufsfeld gegen aussen stärker wahrgenommen, wäre fachlich anerkannter und dadurch auch politisch stärker legitimiert. Unter diesen Umständen wäre es für die Gemeinden einfacher, die Probleme durch Sicherheit mit sozialarbeiterischen Interventionen präventiv anzugehen. Dies wäre ein willkommener Schritt, um das friedliche $\mathrm{Zu}$ sammenleben im öffentlichen Raum mit weniger Regulierung zu ermöglichen und das Gemeinwohl durch gegenseitiges Verständnis und Akzeptanz zu erhöhen.

\section{Literatur}

Gahleitner, S. (2017): Soziale Arbeit als Beziehungsprofession. Bindung, Beziehung und Einbettung professionell ermöglichen. Weinheim, Basel: Beltz.

Hafen, M. (2013): Ethik in Prävention und Gesundheitsförderung. Prävention und Gesundheitsförderung, 8(4): 284-288. www.tinyurl.com/swx6kxo, Zugriff 06.02.2020.

Herriger, N. (2014): Empowerment in der Sozialen Arbeit. Eine Einführung. Stuttgart: Kohlhammer.

Miller, W. R./ Rollnick, S. (2015): Motivierende Gesprächsführung. Freiburg i. B.: Lambertus.

Rogers, C. R. (2017): Der neue Mensch. Stuttgart: Klett-Cotta.

Rosenberg, M. B. (2013): Gewaltfreie Kommunikation. Paderborn: Junfermann.

Scharmer, C. O. (2013): Theorie U. Von der Zukunft her führen: Presencing als soziale Technik. Heidelberg: Carl Auer.

Schulz von Thun, F. (1981): Miteinander reden (Sonderausgabe). Reinbek bei Hamburg: Rowohlt.

Schmocker, B. (2010): Berufskodex Soziale Arbeit Schweiz. Ein Argumentarium für die Praxis der Professionellen. Bern: Avenir Social.

Stadt Luzern (2009): SIP Stadt Luzern. Methodenhandbuch. Luzern (internes Dokument).

Stadt Luzern (2014): Haushalt im Gleichgewicht. Luzern.

Thiersch, H. (2005): Lebensweltorientierte Soziale Arbeit. Aufgaben der Praxis im sozialen Wandel. Weinheim, München: Juventa.

Watzlawik, P./Beavin, J. H./Jackson, D. D. (2011): Menschliche Kommunikation: Formen, Störungen, Paradoxien. Bern: Hans Huber. 
Open Access Dieses Kapitel wird unter der Creative Commons Namensnennung 4.0 International Lizenz (http://creativecommons.org/licenses/by/4.0/deed.de) veröffentlicht, welche die Nutzung, Vervielfältigung, Bearbeitung, Verbreitung und Wiedergabe in jeglichem Medium und Format erlaubt, sofern Sie den/die ursprünglichen Autor(en) und die Quelle ordnungsgemäß nennen, einen Link zur Creative Commons Lizenz beifügen und angeben, ob Änderungen vorgenommen wurden.

Die in diesem Kapitel enthaltenen Bilder und sonstiges Drittmaterial unterliegen ebenfalls der genannten Creative Commons Lizenz, sofern sich aus der Abbildungslegende nichts anderes ergibt. Sofern das betreffende Material nicht unter der genannten Creative Commons Lizenz steht und die betreffende Handlung nicht nach gesetzlichen Vorschriften erlaubt ist, ist für die oben aufgeführten Weiterverwendungen des Materials die Einwilligung des jeweiligen Rechteinhabers einzuholen.

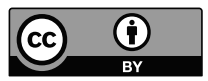

